\title{
Polymorphisms in the CCR5 promoter associated with cervical intraepithelial neoplasia in a Chinese Han population
}

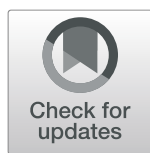

Shuyuan Liu ${ }^{1 \dagger}$, Jun Chen ${ }^{1 \dagger}$, Zhiling Yan², Shuying Dai ${ }^{3,4}$, Chuanyin $\mathrm{Li}^{1}$, Yufeng Yao ${ }^{1 *}$ and Li Shi ${ }^{1 *}$ (D)

\begin{abstract}
Background: C-C chemokine receptor 5 (CCR5) has attracted wide concern for its critical role in the progression of human immunodeficiency virus type 1 (HIV-1) infection. Several studies have demonstrated that CCR5 affects the processes of tumor cell migration, invasion, and metastasis. The aim of this study was to illustrate the association between the polymorphisms of the CCR5 promoter and the development of cervical cancer.

Methods: 336 women with cervical intraepithelial neoplasia (CIN), 488 women with cervical cancer (CC), and 682 healthy controls were recruited to detect polymorphisms in the CCR5 promoter using a sequencing method.

Results: Six loci with polymorphism were found in the CCR5 promoter; the frequencies of the minor alleles of rs1799987 was significantly higher in the CIN group than that in the control group $(P=0.007)$; and the genotypic frequencies of rs2734648, rs1799987, rs1799988 and rs1800023 were significantly different between the CIN group and the control group $(P<0.008)$. The inheritance model analysis showed that rs2734648, rs 1799987, rs1799988 and rs 1800023 significantly increased the susceptibility to CIN in a recessive genetic model $(P<0.008)$. The haplotype constructed by the major alleles of these 6 SNPs (rs2227010-rs1799987-rs1799988-rs2734648-rs1800023-rs1800024: A-G-A-C-A-T) was highly protective against CIN (OR=0.731, 95\%Cl: 0.603-0.886, $P=5.68 \mathrm{E}-03)$. In addition, transcription prediction showed that mutation of these 6 SNPs might alternate the binding of particular transcription factors.
\end{abstract}

Conclusion: The CCR5 promoter polymorphisms were significantly associated with cervical intraepithelial neoplasia by altering the expression of CCR5 on the cell surface in a Chinese Han population.

Keywords: Cervical carcinogenesis, Cervical intraepithelial neoplasia, CCR5 gene, Promoter polymorphism

\section{Background}

C-C motif chemokine receptor type 5 (CCR5), a transmembrane G-coupled cell-surface chemokine receptor, binds to five kinds of CC-chemokines: human macrophage inflammatory protein-1 $\alpha$ (MIP-1 $\alpha$ ), MIP-1 $\beta$, RANTES (regulated on activation, normal $\mathrm{T}$ cell expressed and secreted), monocyte chemotactic protein 2 (MCP-2) and MCP-4 $[1,2]$. CCR5 is known to be the principal coreceptor of macrophage-tropic (R5) strains of human immunodeficiency

\footnotetext{
* Correspondence: leoyyf@gmail.com; shili.imb@gmail.com

†'Shuyuan Liu and Jun Chen contributed equally to this work.

'Institute of Medical Biology, Chinese Academy of Medical Sciences \& Peking Union Medical College, Yunnan Key Laboratory of Vaccine Research \& Development on Severe Infectious Disease, Kunming 650118, Yunnan, China Full list of author information is available at the end of the article
}

virus-type 1 (HIV-1), which is highly variably expressed on the cell surface of the memory $\mathrm{T}$ cells, macrophages, dendritic cells, hematopoietic stem cells, and microglial cells $[1,3]$.

The human CCR5 gene located on 3p21.31, is composed of four exons and two introns. The promoter region has been described previously $[4,5]$, and the differences in the promoter region may regulate the expression of CCR5 in monocyte/macrophages and $\mathrm{T}$ lymphocytes [5]. CCR5 expression in tumor cells and various host cells plays a very important role in tumor progression [6]. Studies have demonstrated that CCR5 and CCR5 ligands CCL5 might stimulate angiogenesis as growth factors, modulate the recruitment of inflammatory cells, and induce the tumor immune evasion $[6,7]$. It has been reported that men with a 
loss of functional CCR5 and carrying the CCR5 $\triangle 32$ mutant are resistant to the development of prostate cancer. Additionally, the expression of the CCR5 ligand CCL5 promotes the migration and invasiveness of pancreatic cancer [8].

Cervical carcinoma is the second most common cancer affecting women worldwide [9]. Approximately 75,000 women develop cervical cancer, and 40,000 women die from this disease in China each year [10]. The development of cervical carcinoma is strongly linked to persistent infection of high-risk human papillomaviruses (HPV), such as HPV16 and HPV18 [11]. Most HPV infections do not cause any symptoms or disease and clear up spontaneously; however, a minority of women exhibit persistent HPV infection, which might lead to cervical intraepithelial neoplasia (CIN) or cervical cancer (CC) $[12,13]$, which means that the host immune system and genetic background play important roles in the progression of cervical cancer [14]. In recent years, Che et al [15] and Sales et al [16] found that CCR5 expression is extremely higher in cervical cancer tissue than in matched normal control tissue and that downregulation of CCR5 suppresses cervical cancer cell growth and proliferation in vivo [15]. Variability of the promoter region of CCR5 gene might be the reason for differing CCR5 expression levels.

In the present study, we detected the genetic polymorphisms in the promoter region of CCR5 of a Chinese Han population and investigated the association between genetic polymorphisms of CCR5 gene promoter and cervical cancer in Han Chinese.

\section{Methods}

\section{Subjects}

A total of 336 cervical intraepithelial neoplasia (CIN) and 488 cervical cancer (CC) female patients were enrolled in the Third Affiliated Hospital of Kunming Medical University (Kunming, China) from 2011 to 2015. All subjects were Han Chinese in Yunnan province (Southwest China).

Diagnoses of CIN and CC were based on comprehensive cervical cancer control guidelines from the World Health Organization (WHO) [17]. Patients undergoing any anti-cancer therapy, such as radiotherapy and chemotherapy before surgery, suffering from other malignant tumors, or with cardiovascular disease, diabetes, hepatitis, kidney disease, etc. were excluded.

In the same period, 682 healthy women were recruited as a control group. The control subjects were healthy women without any history of abnormal Pap Smear or cervical lesions and other cancers on the day of recruitment.

\section{DNA extraction and sequencing}

The genomic DNA of all patients was extracted from the peripheral lymphocytes using the QIAamp Blood Mini Kit (Qiagen, Hilden, Germany).
The CCR5 promoter region was PCR-amplified using the following primers, CCR5P_F: 5' -gacgagaaagctgagggtaaga-3' and CCR5P_R: 5' -taaccgtctgaaactcattcca-3', and PCR fragment was 1388 bp (Fig. 1). PCR for each sample was carried out in a 50- $\mu \mathrm{L}$ reaction volume containing 10 ng of DNA, $10 \mathrm{pmol}$ of each primer, $1.25 \mathrm{U}$ ExTaq polymerase (TaKaRa, Dalian, China), $200 \mathrm{mmol} / \mathrm{L}$ dNTPs $(\mathrm{TaKaRa})$ and $1 \times$ ExTaq PCR buffer (TaKaRa). Amplification consisted of an initial denaturation step of $5 \mathrm{~min}$ at $98^{\circ} \mathrm{C}$; 30 cycles of $10 \mathrm{~s}$ of denaturation at $98^{\circ} \mathrm{C}, 5 \mathrm{~s}$ of annealing at $55^{\circ} \mathrm{C}$, extension at $72{ }^{\circ} \mathrm{C}$ for $90 \mathrm{~s}$; and a final extension for $5 \mathrm{~min}$ at $72^{\circ} \mathrm{C}$. The purified PCR fragment was sequenced to detect the polymorphism loci in this region, using the same primers as in PCR reaction, with Big Dye Terminator Reaction Mix (Applied Biosystems, Foster City, CA, USA). The reaction products were purified by Big Dye XTerminator Purification Kit (Applied Biosystems) and run on ABI 3730XL sequencer. Sequencing results were analyzed using the DNASTAR Lasergene v7.1 package.

\section{Transcription prediction}

PROMO version 8.3 (http://alggen.lsi.upc.es/cgi-bin/promo_ v3/promo/promoinit.cgi?dirDB=TF_8.3) was used to predict the potential binding sites in CCR5 promoter of transcription factors $[18,19]$.

\section{Statistical analysis}

The ages among different groups were compared using one-way ANOVA, followed by an LSD test for multiple comparisons correction. Basic statistical analysis for allele and genotype and disease association was performed using PLINK v1.9 (http://zzz.bwh.harvard.edu/plink/data. shtml) [20].

All polymorphic loci were tested for deviation from the Hardy-Weinberg equilibrium in the control group with a threshold of 0.05 . Association tests for allelic and genotypic frequencies, as well as dominant and recessive inheritance models of these SNPs among CIN, CC and control groups using the $X^{2}$-test were also performed by PLINK. Risks were estimated by the odds ratios (OR) with $95 \%$ confidence interval $(95 \% \mathrm{CI})$. Linkage disequilibrium (LD) among these SNPs was also estimated, where the linkage disequilibrium coefficient $\mathrm{D}$ was calculated using Haploview 4.2 software. LD is displayed as pairwise $\mathrm{D}$, and the $\mathrm{D}^{\prime}$ values defined in the range $[-1,1]$, with a value of 1 representing perfect disequilibrium. The D' value over 0.8 indicated the existence of different loci in the LD. The differences in the haplotypes between the case and control groups were determined with the $X^{2}$-test. Power-analysis was performed using Power and Sample Size Calculations (version 3.1.2) [21]. Significance threshold after Bonferroni correction for multiple comparisons is $P$ value less than 0.008 . 


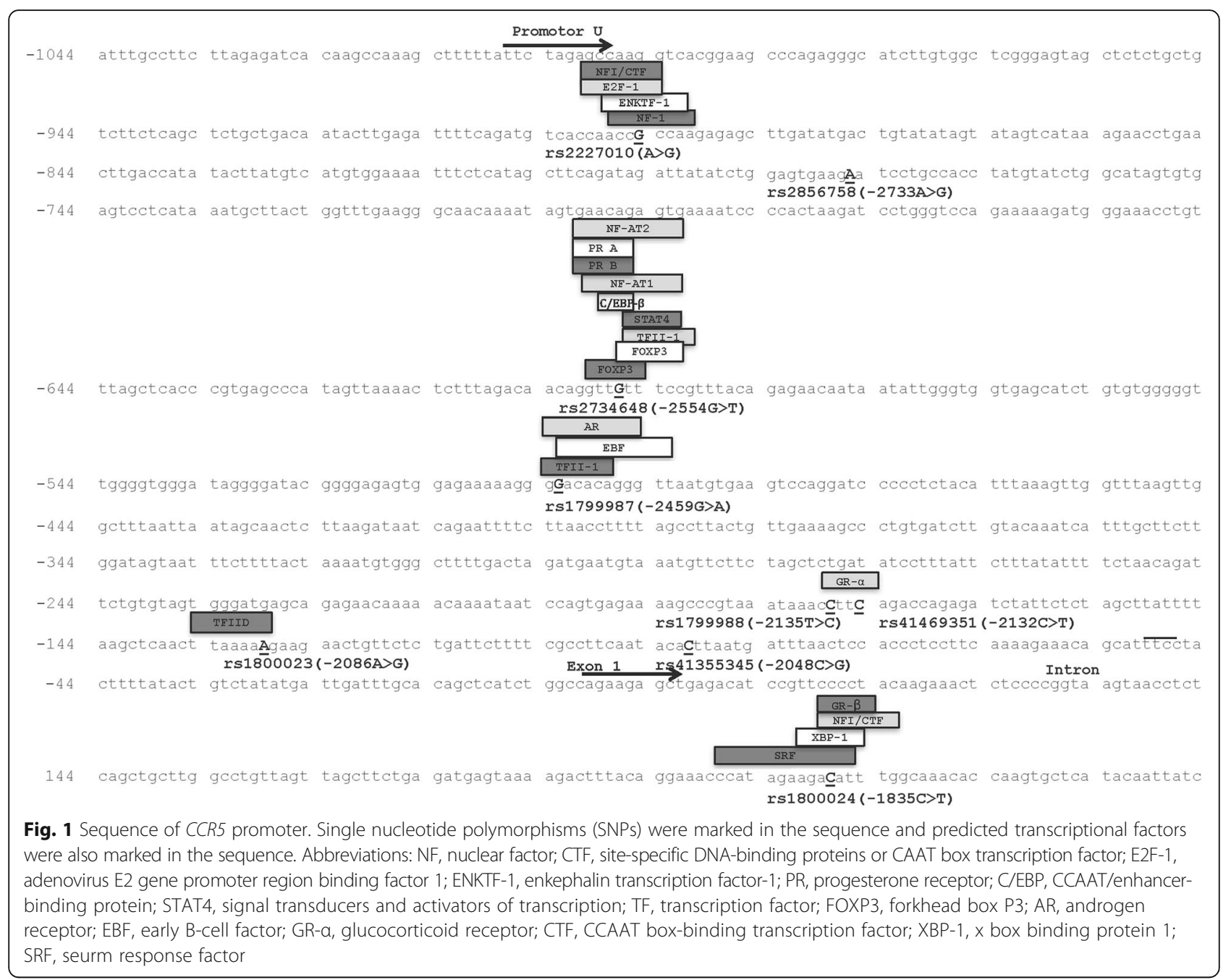

\section{Results}

\section{Characteristics of subjects}

There were 336 CIN patients and 488 CC patients compared with 682 normal women. The mean age was $48.122 \pm 9.578$ (ranged from 22 to 75 ) years in CIN group, $48.180 \pm 9.655$ (ranged from 24 to 73 ) years in the CC group, and $48.934 \pm 7.390$ (ranged from 33 to 79 ) years in the control group. No significant differences in age were found in the pairwise comparisons of $\mathrm{CIN}, \mathrm{CC}$, and control group $(P>0.05)$.

\section{Polymorphism loci in CCR5 promoter region}

According to GenBank (NC_000003.12), there are 9 identical single-nucleotide polymorphism (SNP) loci in the promoter of CCR5 (Fig. 1): rs2227010 (A > G), rs2856758 $(\mathrm{A}>\mathrm{G}), \mathrm{rs} 2734648(\mathrm{~T}>\mathrm{G}), \mathrm{rs} 1799987(\mathrm{G}>\mathrm{A}), \mathrm{rs} 1799988$ ( $\mathrm{T}>\mathrm{C}), \mathrm{rs} 41469351(\mathrm{C}>\mathrm{T}), \mathrm{rs} 1800023(\mathrm{G}>\mathrm{A}), \mathrm{rs} 41355345$ $(\mathrm{C}>\mathrm{G})$ and rs1800024 (C>T). Six SNPs (rs2227010, rs2734648, rs1799987, rs1799988, rs1800023 and rs180024) were found with polymorphism in the Chinese Han population, whereas all participants were AA at rs2856758, CC at rs41469351 and CC at rs41355345.

\section{Association of polymorphism in CCR5 promoter region with cervical cancer}

All these 6 SNPs with polymorphism were in the HardyWeinberg equilibrium (HWE) in the control group $(P>0.05)$. The distribution of allelic and genotypic frequencies of these 6 SNPs among CIN, CC and control group are presented in Tables 1 and 2, the frequencies of the G-allele of rs2734648, were significantly higher in the CIN group than in the control group $(P=0.007, \mathrm{OR}=$ 1.289, 95\%CI: 1.071-1.551, Table 1). The genotypic frequencies of rs2734648, rs1799987, rs1799988 and rs1800023 were distinctly different between the CIN group and the control group $(P<0.008$, Table 2$)$. In addition, no significant differences in allelic or genotypic frequencies were found between the $\mathrm{CC}$ and control groups.

Our study had a power over $80 \%$ to detect and odds ratios (ORs) of 1.403 for rs2227010, 1.971 for rs2734648, 
Table 1 Allelic distribution of SNPs in CCR5 promoter among control, CIN and CC groups

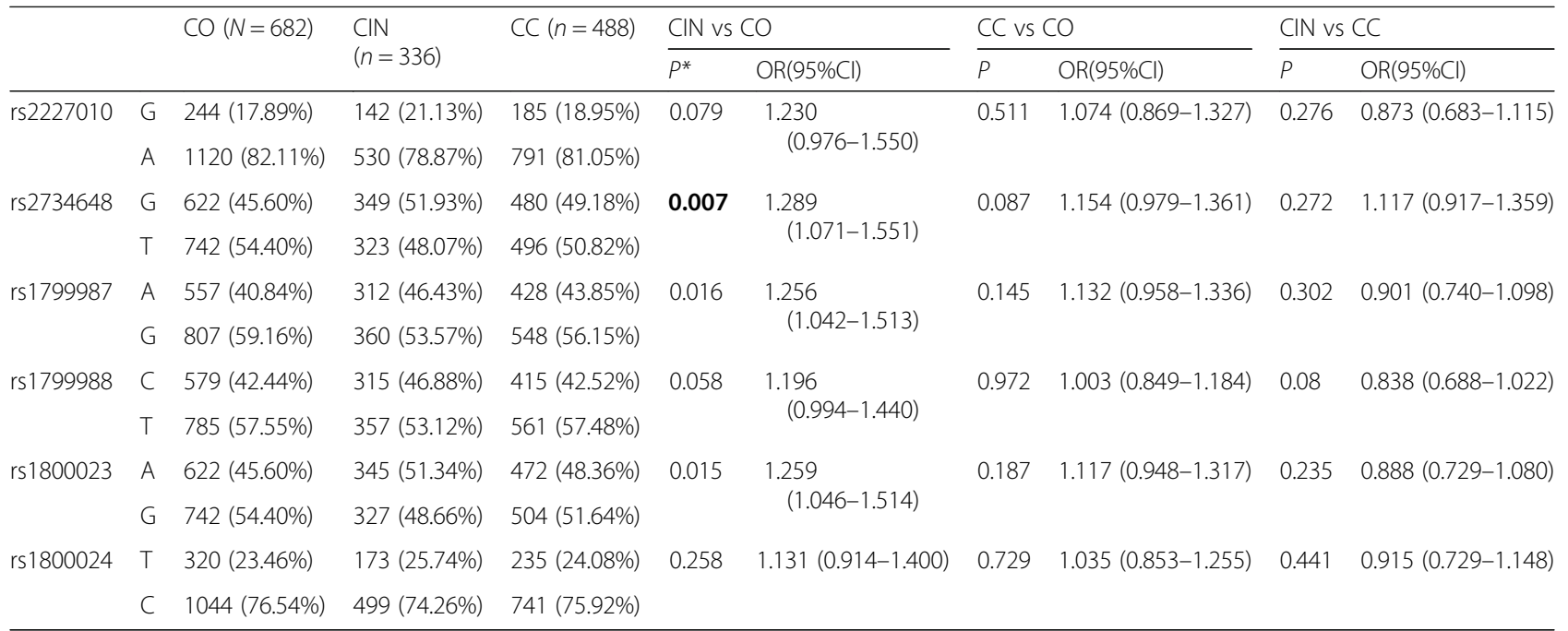

Abbreviations: SNP Single nucleotide polymorphism, CIN Cervical intraepithelial neoplasia, CC Cervical cancer, CO Control, vs Versus, OR Odds ratio, CI Confidence Interval

*Significance threshold after Bonferroni correction for multiple comparisons is $P<0.008$ (which was indicated in bold)

1.963 for rs1799987, 1.898 for rs1799988, 1.906 for rs1800023, and 1.661 for rs1800024, considering the sample size of cases and controls and assuming the minor allele frequencies of each SNP in the Han Chinese population of Yunnan.

\section{Inheritance model analysis among the CIN, CC and control groups}

We also applied dominant and recessive inheritance model analysis to investigate the association between these 6 SNPs in the CCR5 promoter and cervical cancer

Table 2 Genotypic distribution of SNPs in CCR5 promoter among control, CIN and CC groups

\begin{tabular}{|c|c|c|c|c|c|c|c|}
\hline & & Control $(\mathrm{N}=682)$ & $\mathrm{CIN}(\mathrm{n}=336)$ & $C C(n=488)$ & $\mathrm{CIN}$ vs CO & CC vs CO & CIN vs CC \\
\hline \multirow[t]{3}{*}{ rs2227010 } & GG & $28(4.11 \%)$ & $11(3.27 \%)$ & $24(4.92 \%)$ & \multirow[t]{3}{*}{$x^{2}=7.174, P^{*}=0.028$} & \multirow[t]{3}{*}{$X^{2}=0.522, P=0.770$} & \multirow[t]{3}{*}{$X^{2}=6.099, P=0.047$} \\
\hline & GA & $188(27.56 \%)$ & $120(35.72 \%)$ & 137 (28.07\%) & & & \\
\hline & AA & $466(68.33 \%)$ & 205 (61.01\%) & $327(67.01 \%)$ & & & \\
\hline \multirow[t]{3}{*}{ rs 2734648} & GG & $143(20.97 \%)$ & 109 (32.44\%) & $131(26.84 \%)$ & \multirow[t]{3}{*}{$X^{2}=17.260, P=1.78 \mathrm{E}-04$} & \multirow[t]{3}{*}{$X^{2}=5.623, P=0.060$} & \multirow[t]{3}{*}{$X^{2}=3.658, P=0.161$} \\
\hline & TG & $336(49.26 \%)$ & 131 (38.99\%) & $218(44.67 \%)$ & & & \\
\hline & $\pi$ & $203(29.77 \%)$ & $96(28.57 \%)$ & 139 (28.48\%) & & & \\
\hline \multirow[t]{3}{*}{ rs1799987 } & AA & $121(17.74 \%)$ & $93(27.68 \%)$ & $110(22.54 \%)$ & \multirow[t]{3}{*}{$X^{2}=14.59, P=6.78 \mathrm{E}-04$} & \multirow[t]{3}{*}{$X^{2}=4.249, P=0.1195$} & \multirow[t]{3}{*}{$X^{2}=3.420, P=0.181$} \\
\hline & GA & 315 (46.19\%) & $126(37.50 \%)$ & $208(42.62 \%)$ & & & \\
\hline & GG & $246(36.07 \%)$ & 117 (34.82\%) & 170 (34.84\%) & & & \\
\hline \multirow[t]{3}{*}{ rs1799988 } & $\mathrm{CC}$ & $129(18.91 \%)$ & $91(27.08 \%)$ & $96(19.67 \%)$ & \multirow[t]{3}{*}{$X^{2}=9.808, P=0.007$} & \multirow[t]{3}{*}{$X^{2}=0.231, P=0.891$} & \multirow[t]{3}{*}{$x^{2}=6.636, P=0.036$} \\
\hline & $\mathrm{TC}$ & $321(47.07 \%)$ & 133 (39.59\%) & $223(45.70 \%)$ & & & \\
\hline & $\pi$ & $232(34.02 \%)$ & $112(33.33 \%)$ & 169 (34.63\%) & & & \\
\hline \multirow[t]{3}{*}{ rs1800023 } & AA & $147(21.55 \%)$ & 105 (31.26\%) & $127(26.02 \%)$ & \multirow[t]{3}{*}{$X^{2}=11.89, P=0.003$} & \multirow[t]{3}{*}{$x^{2}=3.246, P=0.1973$} & \multirow[t]{3}{*}{$X^{2}=2.904, P=0.234$} \\
\hline & GA & $328(48.10 \%)$ & 135 (40.17\%) & $218(44.67 \%)$ & & & \\
\hline & GG & 207 (30.35\%) & $96(28.57 \%)$ & $143(29.30 \%)$ & & & \\
\hline \multirow[t]{3}{*}{ rs1800024 } & $\pi$ & $43(6.30 \%)$ & $24(7.14 \%)$ & $26(5.33 \%)$ & \multirow[t]{3}{*}{$X 2=1.312, P=0.519$} & \multirow[t]{3}{*}{$X 2=1.510, P=0.470$} & \multirow[t]{3}{*}{$X 2=1.166, P=0.558$} \\
\hline & $C T$ & $234(34.32 \%)$ & 125 (37.21\%) & $183(37.50 \%)$ & & & \\
\hline & $\mathrm{CC}$ & 405 (59.38\%) & 187 (55.65\%) & 279 (57.17\%) & & & \\
\hline
\end{tabular}


development. The results showed that rs2734648, rs1799987, rs1799988 and rs1800023 significantly increased the susceptibility to CIN in the recessive inheritance model $(P<0.008$, Table 3$)$; and rs2734648-GG, rs1799987-AA, rs1799988-CC and rs1800023-AA were the risk genotypes to CIN.

\section{Haplotype analysis among CIN, CC and control group}

The LD among these 6 SNPs was estimated, where the linkage disequilibrium coefficient D (D') was calculated, and the D' value of these 6 SNPs was over 0.8 , which indicated that these 6 SNPs of CCR5 promoter region were in LD.

Then, we constructed the haplotype of these 6 SNPs (rs2227010-rs1799987-rs1799988-rs2734648-rs1800023rs1800024) and pairwise compared the haplotype frequencies among CIN, CC and control groups. Table 4 presents 4 different haplotypes $(\mathrm{H} 1, \mathrm{H} 2, \mathrm{H} 3$ and $\mathrm{H} 4)$ with frequencies over $3 \%$. Haplotype H1 (A-T-G-T-G-C) was the most common haplotype in CIN patients (45.39\%), CC patients (47.54\%) and controls (50.58\%), and the frequency of $\mathrm{H} 1$ was significantly lower in the CIN group compared with the control group $(\mathrm{OR}=0.731,95 \% \mathrm{CI}$ : 0.603-0.886, $P=1.42 \mathrm{E}-03)$. All 4 kinds of haplotypes showed no differences between the $\mathrm{CC}$ and control groups, or between the CIN and CC groups.

\section{Transcription prediction}

PROMO version 8.3 (http://alggen.lsi.upc.es/cgi-bin/promo_ v3/promo/promoinit.cgi?dirDB=TF_8.3) was used to predict the potential binding sites of transcription factors in the CCR5 promoter region [18, 19], the computer algorithms suggested that these 6 SNPs might be binding sites for particular transcription factors (Fig. 1).

\section{Discussion}

Tumor development and progression depend heavily on the presence of angiogenesis factors including chemokines and their receptors [22]. There has been evidence that chemokines and their receptors expressed on tumor cells contribute to recruitment and activation of tumorsuppressor cells $[6,23]$ and recruitment of inflammatory cells to the tumor microenvironment [24], which may induce the process of tumor cell migration, invasion, and metastasis [22, 24-26]. As one of the key chemokines involved in many pathological processes, including inflammation, angiogenesis, tumor cell growth and infiltration, CCR5 may influence the cervical cancer progression. In the current study, we performed the association study of CCR5 promoter $\left(\mathrm{P}_{1}\right)$ polymorphisms with cervical carcinoma and found that the promoter variations of CCR5 might be associated with development of cervical lesions, cervical intraepithelial neoplasia.
Two CCR5 promoters have been previously described: a weak upstream promoter called $\mathrm{P}_{\mathrm{U}}$ or $\mathrm{P}_{2}$, and a stronger downstream promoter called $\mathrm{P}_{\mathrm{D}}$ or $\mathrm{P}_{1}[4,27]$. The expression of CCR5 on cell surface is highly variable, and CCR5 promoter polymorphisms may alter the CCR5 cell surface expression, which might consequently influence the disease progression. Previously, several SNPs in the CCR5 promoter have been shown to affect CCR5 expression. Salkowitz et al found that rs1799987 (-2459G > A) located in the $P_{1}$ was related to a different expression level of CCR5 on $\mathrm{CD}_{1}{ }^{+}$monocytes, and individuals carrying homozygous rs1799987-G exhibited lower CCR5 density on $\mathrm{CD}_{14}{ }^{+}$monocytes [28]. Mummidi et al [29] reported that rs1799987 was associated with the rate of AIDS progression. CCR5 promoter polymorphisms correlate with HIV disease progression possibly by regulating CCR5 cell surface expression and $\mathrm{CD}_{4}{ }^{+} \mathrm{T}$ cell apoptosis in HIV patients. In this study, rs1799987-AA significantly increased the risk of susceptibility to $\mathrm{CIN}$, which indicated that rs1799987-AA might be a risk factor for cervical lesions development, possibly by influencing the expression level of CCR5.

CCR5 promoter haplogroups constructed by $-2733 \mathrm{~A} / \mathrm{G}$, $-2554 \mathrm{G} / \mathrm{T},-2459 \mathrm{G} / \mathrm{A},-2135 \mathrm{~T} / \mathrm{C},-2132 \mathrm{C} / \mathrm{T},-2086 \mathrm{~A} / \mathrm{G}$, $-1835 \mathrm{C} / \mathrm{T}$ and $+554(\Delta 32)$, have been characterized and described before, and CCR5 haplogroups have been named as CCR5-HHA (A-G-G-T-C-A-C- $\Delta 32$ wild), -HHB (A-TG-T-C-A-C- $\Delta 32$ wild), -HHC (A-T-G-T-C-G-C- $\Delta 32$ wild), -HHD (A-T-G-T-T-A-C- $\Delta 32$ wild), -HHE (A-G-A-C-C-AC- $\Delta 32$ wild), -HHF (A-G-A-C-C-A-T- $\Delta 32$ wild), -HHG*1 (G-G-A-C-C-A-C- $\Delta 32$ wild) and -HHG*2 (G-G-A-C-C-AC- $\triangle 32$ mutant) [27, 30, 31]. A specific CCR5 promoter haplogroup was demonstrated to correlate with transcriptional activity [27] and affect the rate of AIDS progression [30]. CCR5-HHA haplotype is associated with lower CCR5 expression and protective phenotype in humans, whereas CCR5-HHF or CCR5-HHG is related to higher CCR5 expression [32]. We performed haplotype analysis with 6 polymorphism loci in CCR5 promoter, and only SNP rs2227010 was not comprised in the defined CCR5 haplogroups. Our results showed that haplotype H1: A-TG-T-G-C, which was similar to CCR5-HHC, was the mostcommon haplotype in our participants and consisted of the major alleles of these 6 SNPs revealing a protective effect against $\mathrm{CIN}$; and a tendency of susceptibility to CIN appeared in H2 (similar to CCR5-HHE), H3 (similar to CCR5-HHF) and H4 (similar to CCR5-HHA), although these haplotypes did not show statistical association with $\mathrm{CIN}$. The haplotype analysis results coincided with the results of our allelic and genotypic analysis and indicated that the CCR5 promoter haplotype was associated with cervical lesions development. However the correlation between haplotype of 6 SNPs in this study and expression of CCR5 need to test and verify. 


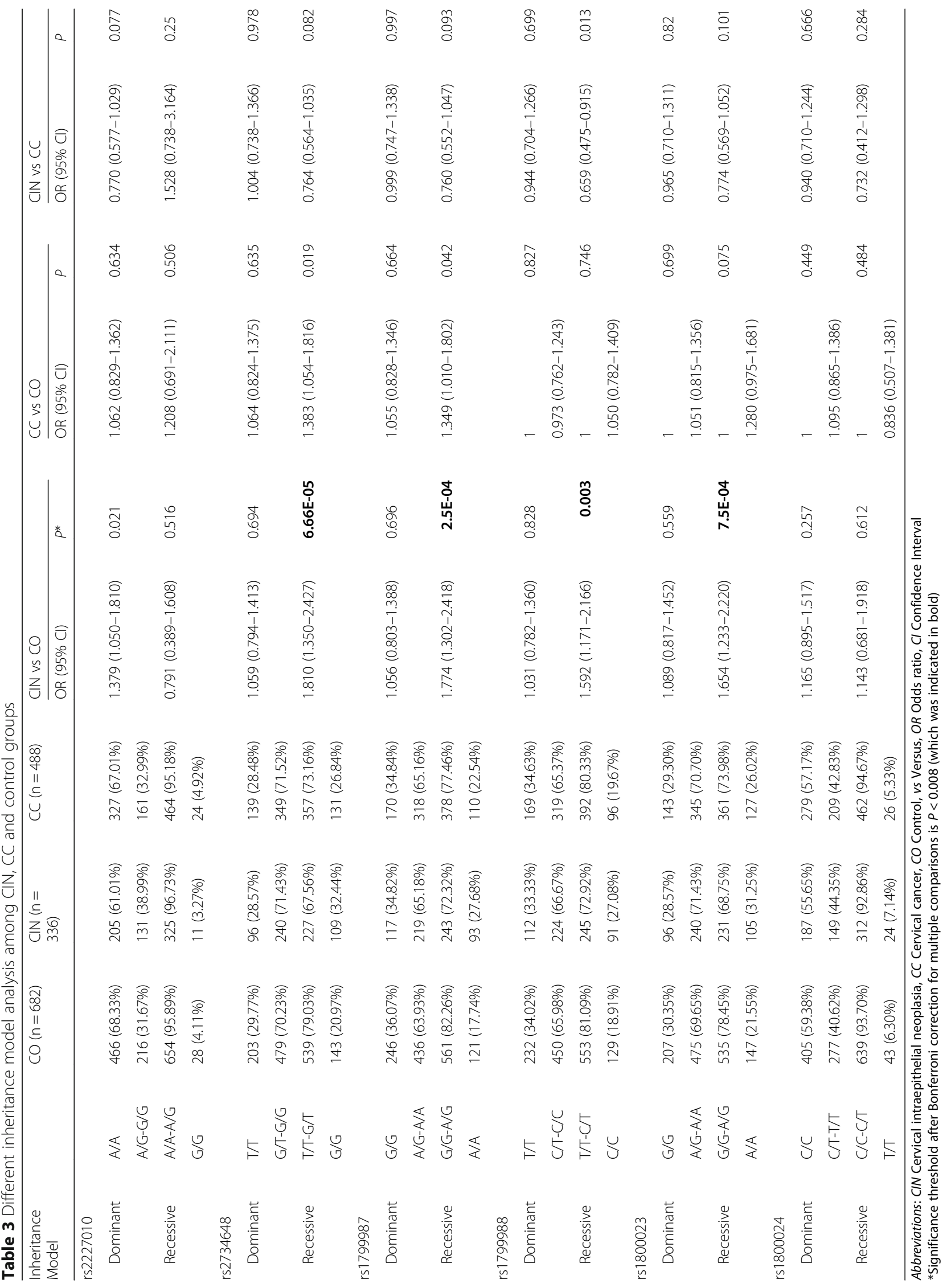




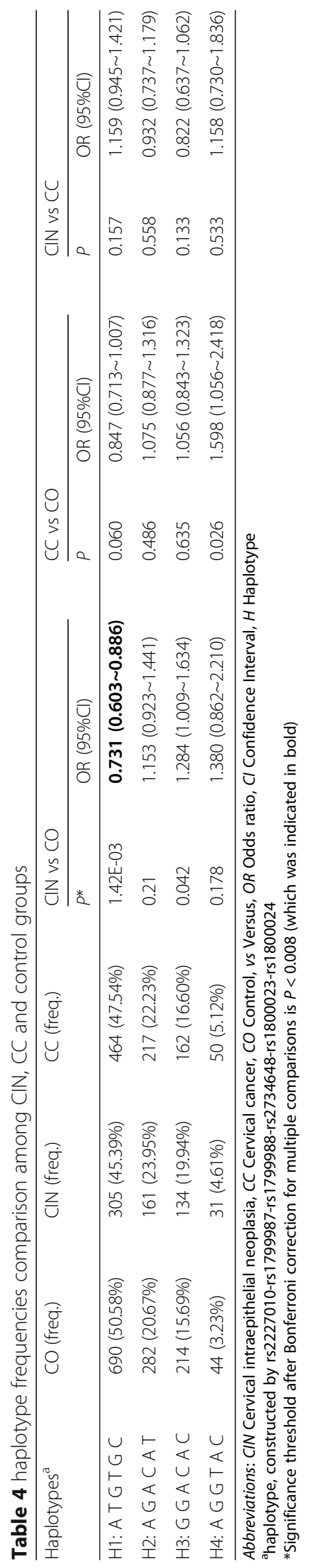


In 2016, Che et al indicated that CCR5 mRNA and protein expression levels were extremely higher in cervical cancer tissue than in matched normal control tissue, and downregulation of CCR5 inhibited cervical cancer growth and invasion [15]. Sales et al also presented similar results that the level of CCR 5 was high in cervical cancer tissue and the expression of CCR5 could be regulated by inflammatory pathways [16]. However, no previous reports on the CCR5 expression level in CIN tissues were identified. So we performed the transcription factor binding site prediction in the CCR5 promoter and try to find the possible mechanism of association of SNPs to development of cervical lesions. The computer algorithms suggested that there are many binding sites of distinct nuclear and transcription factors in the CCR5 promoter, and the 6 SNPs studied were located in the binding region of specific transcription factors (Fig. 1). Nucleotide substitutions in the cis-regulatory region of a gene may cause a loss of binding of some nuclear proteins, or binding of some novel nuclear factors, or alteration in nuclear factor binding affinity. A previous study found that SNPs in human CCR5 cisregulatory sequences were associated with alteration in DNA/nuclear factor binding. Mummidi et al found both $\mathrm{G}$ and $\mathrm{T}$ in -2554 site (rs2734648) could bind to four nuclear factor complexes (NF4-NF7), but the affinities for NF5-NF4 complexes bound to the $-2554 \mathrm{~T}$ were significantly greater than the binding affinities to $-2554 \mathrm{G}$ [27]. Bream et al [33] also found that $-2554 \mathrm{~T}$ bound p65, c-Rel, and p50 with greater affinity than -2554G. We predicted that $\mathrm{rs} 2734648-\mathrm{T}$ was located in the binding region of FOXP3, TFII-I, C/EBP-beta, NF-AT1 and PR-B. Therefore, the transcriptional status of rs2734648-G, especially rs2734648-GG, might be different. So we infer that rs2734648-GG is significantly associated with the susceptibility of CIN by affecting the transcription factors binding. Mummidi et al also found the wild-type $C$ allele in - 1835 site (rs1800024) specifically bound to two novel nuclear factors NF2 and NF3, but the mutant-T allele lost the ability to bind nuclear factor NF3 [27]. The binding region of transcription factor GR- $\alpha$ contains two polymorphic loci rs1799988 $(-2135 \mathrm{~T}>\mathrm{C})$ and rs41469351 $(-2132 \mathrm{C}>\mathrm{T})$. These two polymorphisms are located so closely, only at a 2-base-pair interval. For there is no polymorphism in rs41469351, so nucleotide substitution in rs1799988 might affect the transcription factor GR- $\alpha$ binding. In addition, Mummidi et al [27] found that CCR5 haplotypes conferred the haplotypespecific differences in the promoter transcriptional efficiency, of which the haplotype HHA showed the least promoter activity, whereas the transcriptional activity of HHF haplotype was the highest. That is to say the haplotype $\mathrm{H} 1$, similar to $\mathrm{HHC}$, have a medium promoter activity. And haplotype $\mathrm{H} 1$ showed a significant resistance to CIN. So we infer that nucleotide substitutions such as rs2734648-GG, rs1799987-AA, rs1799988-CC and rs1800023 could associated with susceptibility to CIN by alternate nuclear factors binding,, then subsequently affect the efficiency of CCR5 translation and CCR5 expression level on cell surface and, finally, affect the development of cervical lesions. By the way, our results revealed that the susceptibility of SNPs in CCR5 promoter arising more in recessive inheritance model, such as rs2734648, rs1799987, rs1799988 and rs1800023. That is to say individuals carried two mutations in both alleles raise the susceptibility to CIN. This can be understood as the efficiency of nuclear factors binding, CCR5 translation and expression could be affected more when two mutations in both alleles of SNP.

In this study, we found the frequencies of the minor alleles of rs2734648 was significantly higher in the CIN group than that in the control group and the frequencies of rs2734648-GG, rs1799987-AA, rs1799988-CC and rs1800023-AA were distinctly increase the susceptibility of CIN. However, rare associations of susceptible to CC or from CIN progressing to $\mathrm{CC}$ were found. It is known that persisting high-risk HPV infection, which usually related to the lack of HPV-specific T-cell immunity and some immune-response pathway [11, 13], is associated with the development of vassal cell layer infection and hyperplastic lesions called cervical intraepithelial neoplasia (CIN). CIN lesions may regress, persist or progress to invasive cervical carcinogenesis [14]. Carcinoma development depends on different strategies to escape recognition by immune cells. So we think the mechanisms of normal cervical tissue progressing to CIN and CIN progressing to CC might be different, and the host immunogenetic factors played in development and progression of cervical cancer need to further study.

\section{Conclusion}

In conclusion, we demonstrated that SNPs in the CCR5 promoter are significantly associated with development of cervical intraepithelial neoplasia. These polymorphisms could potentially influence not only the CCR 5 gene expression but also the CCR5 mRNA stability and the translation efficiency, which subsequently influence the density of CCR5 on cell-surface and, thus, have an impact on virus infection or disease pathogenesis. However, there might be some other mechanism of cervical cancer development, because our results indicated that polymorphisms in the CCR5 promoter are not associated with progression from CIN to CC. Thus, sample size expansion for association study and the functional study on correlation of CCR5 polymorphisms with cervical intraepithelial neoplasia development, and finding other immunogenetic factors associated with cervical carcinoma will be performed in the future. 


\section{Abbreviations}

AR: Androgen receptor; C/EBP: CCAAT/enhancer-binding protein; CC: Cervical cancer; CCR5: C-C motif chemokine receptor type 5; CIN: Cervical intraepithelial neoplasia; CTF: CCAAT box-binding transcription factor; CTF: Site-specific DNA-binding proteins or CAAT box transcription factor; E2F-1: Adenovirus E2 gene promoter region binding factor 1; EBF: Early B-cell factor; ENKTF-1: Enkephalin transcription factor-1; FOXP3: Forkhead box P3; GR-a: Glucocorticoid receptor; HIV: Human immunodeficiency; HPV: Human papillomaviruses; HWE: Hardy-Weinberg equilibrium; LD: Linkage disequilibrium; MCP: Monocyte chemotactic protein; MIP: Macrophage inflammatory protein; NF: Nuclear factor; OR: Odds ratio; PR: Progesterone receptor; RANTES: Regulated on activation, normal T cell expressed and secreted; SNP: Single-nucleotide polymorphism; SRF: Serum response factor; STAT4: Signal transducers and activators of transcription; TF: Transcription factor; XBP-1: $X$ box binding protein 1

\section{Acknowledgments}

We are grateful to patients and healthy control individuals who participated in this study.

\section{Authors' contributions}

The authors SYL and JC finished main part experiment and data analysis; ZLY finished the sample clinical diagnose and collection; SYL, YFY and LS drafted this manuscript; SYD and CYL did the DNA extraction and part of PCR amplification experiment; LYY did part of the data collection and analysis; YFY and LS designed the project and reviewed the manuscript; SYL, ZLY, SYD, YFY and LS acquired the fundings. And all authors have read and approved the manuscript.

\section{Funding}

This work was supported by grants from the National Natural Science Foundation of China (31401063 and 81573206), Applied Basic Research Projects of Yunnan province (2016FA034, 2017FE467-012), the PUMC Youth Fund (3332015149), Special Funds for high-level health talents of Yunnan Province (D-201669 and L-201615) and CAMS Innovation Fund for Medical Science (2016-12 M-2-001). The funders had no role in study design, data collection and analysis, decision to publish, or preparation of the manuscript.

\section{Availability of data and materials}

All data generated or analyzed during this study are available to any scientist wishing to use them for non-commercial purposes from the corresponding author on reasonable request. But those clinical data are not available for authors have an ethical and legal responsibility to respect participant's rights to privacy and to protect their identity.

\section{Ethics approval and consent to participate}

All experimental protocols used in this study were approved by the Institutional Review Board of the Third Affiliated Hospital of Kunming Medical University (Kunming, China). All procedures were in accordance with the approved guidelines and principles expressed in the Helsinki Declaration and its later amendments or comparable ethical standards of 1975. And all participants provided written informed consent.

\section{Consent for publication}

Not applicable.

\section{Competing interests}

The authors declare that they have no competing interests.

\section{Author details}

${ }^{1}$ Institute of Medical Biology, Chinese Academy of Medical Sciences \& Peking Union Medical College, Yunnan Key Laboratory of Vaccine Research \& Development on Severe Infectious Disease, Kunming 650118, Yunnan, China. ${ }^{2} T$ The Third People's Hospital of Kunming, Kunming 650041, China. ${ }^{3}$ School of Basic Medical Science, Kunming Medical University, Kunming 650500, China. ${ }^{4}$ School of Pharmaceutical Science, Yunnan Key Laboratory of Pharmacology for Natural Products, Kunming Medical University, Kunming 650500, China.
Received: 13 February 2019 Accepted: 21 May 2019

Published online: 31 May 2019

\section{References}

1. Blanpain C, Lee B, Tackoen M, Puffer B, Boom A, Libert F, Sharron M Wittamer V, Vassart G, Doms RW, et al. Multiple nonfunctional alleles of CCR5 are frequent in various human populations. Blood. 2000;96(5):1638-45.

2. Blanpain C, Migeotte I, Lee B, Vakili J, Doranz BJ, Govaerts C, Vassart G, Doms RW, Parmentier M. CCR5 binds multiple CC-chemokines: MCP-3 acts as a natural antagonist. Blood. 1999;94(6):1899-905.

3. He J, Chen Y, Farzan M, Choe H, Ohagen A, Gartner S, Busciglio J, Yang X, Hofmann W, Newman W, et al. CCR3 and CCR5 are co-receptors for HIV-1 infection of microglia. Nature. 1997;385(6617):645-9.

4. Mummidi S, Ahuja SS, McDaniel BL, Ahuja SK. The human CC chemokine receptor 5 (CCR5) gene. Multiple transcripts with $5^{\prime}$-end heterogeneity, dual promoter usage, and evidence for polymorphisms within the regulatory regions and noncoding exons. J Biol Chem. 1997;272(49):30662-71.

5. Guignard F, Combadiere C, Tiffany HL, Murphy PM. Gene organization and promoter function for CC chemokine receptor 5 (CCR5). J Immunol (Baltimore, Md : 1950). 1998;160(2):985-92.

6. Umansky V, Blattner C, Gebhardt C, Utikal J. CCR5 in recruitment and activation of myeloid-derived suppressor cells in melanoma. Cancer Immunol, Immunother : CII. 2017;66(8):1015-23.

7. Aldinucci D, Colombatti A. The inflammatory chemokine CCL5 and cancer progression. Mediat Inflamm. 2014;2014:292376.

8. Singh SK, Mishra MK, Eltoum IA, Bae S, Lillard JW Jr, Singh R. CCR5/CCL5 axis interaction promotes migratory and invasiveness of pancreatic cancer cells. Sci Rep. 2018;8(1):1323.

9. Mirabello L, Clarke MA, Nelson CW, Dean M, Wentzensen N, Yeager M, Cullen M, Boland JF, Schiffman M, Burk RD. The intersection of HPV epidemiology, genomics and mechanistic studies of HPV-mediated carcinogenesis. Viruses. 2018;10(2):E80. https://doi.org/10.3390/v10020080. https://www.mdpi.com/1999-4915/10/2/80.

10. Arbyn M, Walker A, Meijer CJ. HPV-based cervical-cancer screening in China. Lancet Oncol. 2010;11(12):1112-3.

11. Kim TJ, Jin H-T, Hur S-Y, Yang HG, Seo YB, Hong SR, Lee C-W, Kim S, Woo JW, Park KS, et al. Clearance of persistent HPV infection and cervical lesion by therapeutic DNA vaccine in CIN3 patients. Nat Commun. 2014;5:5317.

12. Ljubojevic S, Skerlev M. HPV-associated diseases. Clin Dermatol. 2014;32(2):227-34

13. Tindle RW. Immune evasion in human papillomavirus-associated cervical cancer. Nat Rev Cancer. 2002;2:59.

14. de Freitas AC, Gurgel APAD, Chagas BS, Coimbra EC, do Amaral CMM. Susceptibility to cervical cancer: an overview. Gynecol Oncol. 2012;126(2):304-11.

15. Che LF, Shao SF, Wang LX. Downregulation of CCR5 inhibits the proliferation and invasion of cervical cancer cells and is regulated by microRNA-107. Exp Ther Med. 2016;11(2):503-9.

16. Sales KJ, Adefuye A, Nicholson L, Katz AA. CCR5 expression is elevated in cervical cancer cells and is up-regulated by seminal plasma. Mol Hum Reprod. 2014;20(11):1144-57.

17. World Health Organization aDoRHaR, and Department of Chronic Diseases and Health Promotion: Comprehensive cervical cancer control: A guide to essential practice. 2006.

18. Messeguer X, Escudero R, Farre D, Nunez O, Martinez J, Alba MM. PROMO: detection of known transcription regulatory elements using species-tailored searches. Bioinformatics (Oxford, England). 2002;18(2):333-4.

19. Farre D, Roset R, Huerta M, Adsuara JE, Rosello L, Alba MM, Messeguer X. Identification of patterns in biological sequences at the ALGGEN server: PROMO and MALGEN. Nucleic Acids Res. 2003;31(13):3651-3.

20. Purcell S, Neale B, Todd-Brown K, Thomas L, Ferreira MA, Bender D, Maller J, Sklar P, de Bakker PI, Daly MJ, et al. PLINK: a tool set for whole-genome association and population-based linkage analyses. Am J Hum Genet. 2007:81(3):559-75.

21. Dupont WD, Plummer W. Power and sample size calculations, A Review and Computer Program, vol. 11; 1990

22. Rossi D, Zlotnik A. The biology of chemokines and their receptors. Annu Rev Immunol. 2000;18(1):217-42.

23. Schlecker E, Stojanovic A, Eisen C, Quack C, Falk CS, Umansky V, Cerwenka A. Tumor-infiltrating monocytic myeloid-derived suppressor cells mediate 
CCR5-dependent recruitment of regulatory T cells favoring tumor growth. J Immunol (Baltimore, Md : 1950). 2012;189(12):5602-11.

24. Razmkhah M, Arabpour F, Taghipour M, Mehrafshan A, Chenari N, Ghaderi A. Expression of chemokines and chemokine receptors in brain tumor tissue derived cells. Asian Pac J Cancer Prev : APJCP. 2014;15(17):7201-5.

25. Youngs SJ, Ali SA, Taub DD, Rees RC. Chemokines induce migrational responses in human breast carcinoma cell lines. Int J Cancer. 1997;71(2):257-66.

26. Kleeff J, Kusama T, Rossi DL, Ishiwata T, Maruyama H, Friess H, Buchler MW, Zlotnik A, Korc M. Detection and localization of Mip-3alpha/LARC/Exodus, a macrophage proinflammatory chemokine, and its CCR6 receptor in human pancreatic cancer. Int J Cancer. 1999;81(4):650-7.

27. Mummidi S, Bamshad M, Ahuja SS, Gonzalez E, Feuillet PM, Begum K, Galvis MC, Kostecki V, Valente AJ, Murthy KK, et al. Evolution of human and nonhuman primate $\mathrm{CC}$ chemokine receptor 5 gene and mRNA. Potential roles for haplotype and mRNA diversity, differential haplotype-specific transcriptional activity, and altered transcription factor binding to polymorphic nucleotides in the pathogenesis of HIV-1 and simian immunodeficiency virus. J Biol Chem. 2000;275(25):18946-61.

28. Salkowitz JR, Bruse SE, Meyerson H, Valdez H, Mosier DE, Harding CV, Zimmerman PA, Lederman MM. CCR5 promoter polymorphism determines macrophage CCR5 density and magnitude of HIV-1 propagation in vitro. Clin Immunol (Orlando, Fla). 2003;108(3):234-40.

29. McDermott DH, Zimmerman PA, Guignard F, Kleeberger CA, Leitman SF, Murphy PM. CCR5 promoter polymorphism and HIV-1 disease progression. Multicenter AIDS cohort study (MACS). Lancet (London, England). 1998;352(9131):866-70.

30. Martin MP, Dean M, Smith MW, Winkler C, Gerrard B, Michael NL, Lee B, Doms RW, Margolick J, Buchbinder S, et al. Genetic acceleration of AIDS progression by a promoter variant of CCR5. Science (New York, NY). 1998;282(5395):1907-11.

31. Picton AC, Paximadis $M$, Tiemessen $C T$. Genetic variation within the gene encoding the HIV-1 CCR5 coreceptor in two south African populations. Infect Genet Evol : journal of molecular epidemiology and evolutionary genetics in infectious diseases. 2010;10(4):487-94.

32. Gornalusse GG, Mummidi S, Gaitan AA, Jimenez F, Ramsuran V, Picton A, Rogers K, Manoharan MS, Avadhanam N, Murthy KK, et al. Epigenetic mechanisms, T-cell activation, and CCR5 genetics interact to regulate T-cell expression of CCR5, the major HIV-1 coreceptor. Proc Natl Acad Sci U S A. 2015;112(34):E4762-71.

33. Bream JH, Young HA, Rice N, Martin MP, Smith MW, Carrington M, O'Brien SJ. CCR5 promoter alleles and specific DNA binding factors. Science (New York, NY). 1999;284(5412):223.

\section{Publisher's Note}

Springer Nature remains neutral with regard to jurisdictional claims in published maps and institutional affiliations.

Ready to submit your research? Choose BMC and benefit from:

- fast, convenient online submission

- thorough peer review by experienced researchers in your field

- rapid publication on acceptance

- support for research data, including large and complex data types

- gold Open Access which fosters wider collaboration and increased citations

- maximum visibility for your research: over $100 \mathrm{M}$ website views per year

At BMC, research is always in progress.

Learn more biomedcentral.com/submissions 